\title{
Numerical modelling of a coaxial Stirling pulse tube cryocooler with an active displacer for space applications
}

\author{
Hannah Rana*, Mohammad Amin Abolghasemi, Richard Stone, Mike Dadd, Paul \\ Bailey \\ Department of Engineering Science, University of Oxford, UK
}

\begin{abstract}
A numerical model for a coaxial Stirling pulse tube cryocooler with an active displacer has been developed. An active displacer, in place of an inertance tube, has already demonstrated good efficiency in an in-line pulse tube, but incorporating this design into a coaxial configuration permits better access to the cold head. A model of the coaxial cold head was developed to include the radial flow and cooling in this region. The subassembly models were validated with flow testing of the physical sub-units of the cryocooler. The performance has been predicted for the cryocooler as a function of: fill pressure, operating frequency, and phase angle between the position of the linear compressor and displacer. The projected difference in performance and efficiency of the coaxial configuration was compared to the in-line design. The coaxial cryocooler numerically simulates $6 \mathrm{~W}$ of cooling at $80 \mathrm{~K}$ with an input power of $85 \mathrm{~W}$, at a fill pressure of $28 \mathrm{bar}$, an operating frequency of $60 \mathrm{~Hz}$, and a compressor-displacer phase angle of $41^{\circ}$. Overall, the coaxial cryocooler outperforms the in-line design in terms of cooling power, but not in terms of efficiency.
\end{abstract}

Keywords: Cryocooler; Pulse tube; Stirling cycle; Refrigerator; Cooling power; Active displacer; Coaxial pulse tube; Sage numerical modelling

\begin{tabular}{|cc|}
\hline $\begin{array}{c}\text { Nomenclature } \\
\text { COMP }\end{array}$ & Compressor \\
\hline
\end{tabular}

\footnotetext{
* Corresponding author.

Email address: hannah.rana@eng.ox.ac.uk
} 


\begin{tabular}{|cl|}
\hline DISP & Displacer \\
FS & Flow straightener \\
PT & Pulse tube \\
CHX & Cold end heat exchanger \\
WHX & Warm end heat exchanger \\
REG & Regenerator \\
$Q_{c}$ & Cooling power (W) \\
$P_{1}$ & Pressure of the gas after compression \\
$P_{2}$ & Pressure of the gas exiting the warm end heat exchanger \\
$\eta_{r}$ & Relative Carnot efficiency \\
$W_{s h}$ & Shaft power (W) \\
$T_{h}$ & Warm end temperature (K) \\
$T_{c}$ & Cold end temperature (K) \\
pk-pk & Peak-to-peak \\
$\Phi_{p, P T}$ & Phase angle between pressure pulses either side of pulse tube $\left({ }^{\circ}\right)$ \\
$\Phi_{p, R E G}$ & Phase angle between pressure pulse either side of regenerator $\left(^{\circ}\right)$ \\
$\Delta \mathrm{P}_{p k-p k, P T}$ & Difference in peak-to-peak pressure across pulse tube (bar) \\
$\Delta \mathrm{P}_{p k-p k, R E G}$ & Difference in peak-to-peak pressure across regenerator (bar) \\
$\Phi m_{, P T}$ & Phase angle in mass flow either side of pulse tube $\left(^{\circ}\right)$ \\
$\Delta m_{p k-p k, P T}$ & Difference in peak-to-peak mass flow either side of pulse tube $(\mathrm{g} / \mathrm{s})$ \\
$\Phi m_{, R E G}$ & Phase angle in mass flow across regenerator $\left(^{\circ}\right)$ \\
$\Delta m_{p k-p k, R E G}$ & Difference in peak-to-peak mass flow across regenerator $(\mathrm{g} / \mathrm{s})$ \\
\hline &
\end{tabular}

\section{Introduction}

Stirling pulse tube cryocoolers (SPTCs) provide active cooling for superconducting and infrared technology on board spacecraft. A pulse tube requires a well-designed interplay between the pressure pulse carried throughout the cryocooler and the mass flow. Orifices and inertance tubes are commonly used to achieve this [1], however they 
do not allow for the control and adjustment of the pressure pulse. An active displacer has also been demonstrated to attain such a relationship between these two parameters, with additional benefits that potentially lead to a higher efficiency [2]. A warm end active displacer is able to effectively control and achieve the correct relationship between the mass flow and pressure at the cold end more readily than orifices and inertance tubes [3]. Furthermore, the expansion power at the pulse tube warm end can be recovered through the displacer, resulting in principle to a more efficient SPTC [4].

The advantage of operating the cryocooler in a coaxial configuration is the reduction of volume and enhanced ease of integration between the cold head and the cooling interface [5] [6] [7]. Hence, it is of great practical use to determine the effectiveness of integrating an efficiency-enhancing active displacer into an SPTC in a coaxial configuration.

A coaxial pulse tube using an active displacer has been designed within the Cryogenic Engineering group at the University of Oxford in collaboration with Honeywell Hymatic. This paper outlines the numerical modelling approaches and subassembly model validation that was completed in order to shed light on the effectiveness of the cryocooler. Sage software [8] [9] was utilised to devise a numerical model that represents the thermodynamic activity within the cryocooler. The design of the cryocooler is discussed in Section 2, followed by the modelling approaches and results of the model validation at a sub-assembly level in Section 3. The performance predictions for the cryocooler are presented in Section 4 along with sensitivity analyses and a comparison with the in-line cryocooler performance [2].

\section{In-line and Coaxial Cryocooler Design}

The pulse tube configurations are shown in Figure 1. The compression unit, consisting of two pistons, connects to the displacer as well as the heat rejector at the bottom of the annular regenerator. In the coaxial configuration, the pulse tube is located inside in the annular regenerator. The pulse tube connects back to the displacer unit. 

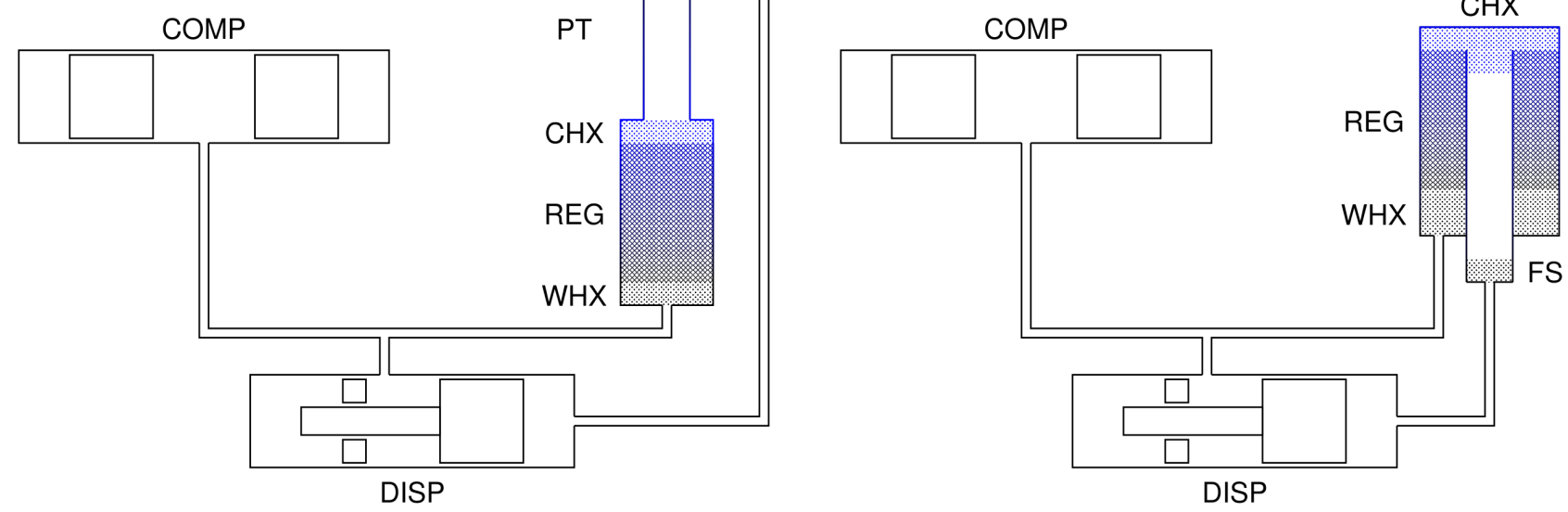

Figure 1: Schematic of (left) the in-line cryocooler with an active displacer and 60 (right) a coaxial cryocooler with an active displacer. COMP is the compression unit, DISP is the displacer, WHX is the warm end heat exchanger (i.e. heat rejector), PT is the pulse tube, CHX represents the cold head heat exchanger, REG is the regenerator, and FS is the flow straightener.

65 Table 1: Dimensions of the pulse tube and regenerator in the in-line and coaxial configurations of the cryocooler design.

\begin{tabular}{c|cc|ccc}
\hline \multirow{2}{*}{ Configuration } & \multicolumn{2}{|c|}{ Pulse Tube } & \multicolumn{3}{c}{ Regenerator } \\
\cline { 2 - 6 } & Length $(\mathrm{mm})$ & $\begin{array}{c}\text { Internal } \\
\text { Diameter }(\mathrm{mm})\end{array}$ & Length $(\mathrm{mm})$ & Diameter $(\mathrm{mm})$ & $\begin{array}{c}\text { Cross- } \\
\text { Sectional Area } \\
\left(\mathrm{mm}^{2}\right)\end{array}$ \\
\hline In-Line & 85 & 7.7 & 46.3 & 15.5 & 189 \\
Coaxial & 91 & 7.9 & 60 & 11.1 (ID) $/ 18.5$ & 173 \\
\hline
\end{tabular}

\section{Numerical Simulation}

There are several key parameters that affect the numerical modelling of the 
model for the heat exchanger mesh, the mesh block porosity, wire diameter, and block diameter were experimentally investigated on prototype sub-assemblies to select optimal values which inspired inputs for the numerical model. Porosity of the mesh block is difficult to estimate, where a rough approximation is obtained by calculating the void fraction using the ratio between the wire volume and the block volume. This can alter significantly once the mesh block is packed, hence a numerical optimal porosity value must be calculated for which a minimum error between the numerical model output and the flow tests occurs. Flow tests using a mass flow meter were completed on the prototype heat rejector block (shown in Figure 2) and coaxial cold head physical sub-assemblies (Figure 3) to provide confidence for realistic values of porosity and effective lengths of gas flow to be put into Sage. $\mathrm{P}_{2}$ is at atmospheric pressure and a pressure transducer was used at $\mathrm{P}_{1}$ to measure the pressure drop. The porosity was originally estimated as 0.67 but the flow test validated value was found to be 0.65 . This was then used as a more representative input for the numerical model.

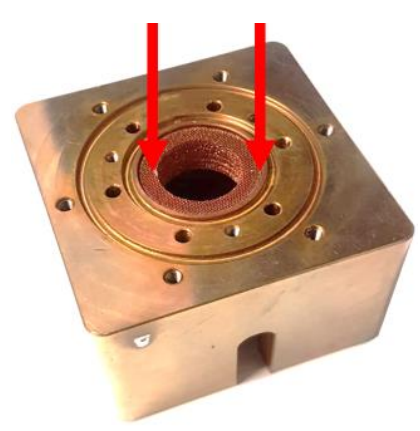

85

Figure 2: The copper block heat rejector with arrows indicating the annular flow through the mesh (left) and the flow test setup through the block measuring the pressure drop across the system (right). The yellow central cylinder indicates the aluminium used uniquely in the flow test setup, in the place of the pulse tube in the cryocooler.

90

In the coaxial cold head, as the gas flow changes course and direction from one axis to another, it is difficult to identify the effective gas flow length and diameter, as depicted in Figure 3 (top right). Furthermore, given the fact that radial flow for the gas may be easier than axial flow in the block of layers of woven screen mesh, the one 
dimensional isotropic numerical model that Sage permits is limited, which would yield higher pressure drops than those observed in reality when comparing pressure drop in the flow tests with those numerically computed. The effective length of the gas flow through the coaxial cold head is found to be $70 \%$ of the physical prototype length (see Table 1), which was determined by minimizing the RMS error between the model and the flow test pressure drop across the cold head (Figure 3). It is important to note that the sub-model is validated purely based on pressure drop, and is specific to the particular geometry. The effective parameters derived for this particular heat exchanger cannot be readily used for a different component unless key dimensionless parameters are equivalent. Furthermore, the heat transfer due to radial mixing of the gas in this region is not accounted for, and this would require more detailed fluid simulation for further accuracy. Zhao et al. developed a CFD model of a miniature coaxial SPTC with focus on gas and solid conduction in the regenerator, and reported significant losses [10] compared to one dimensional numerical simulation results.

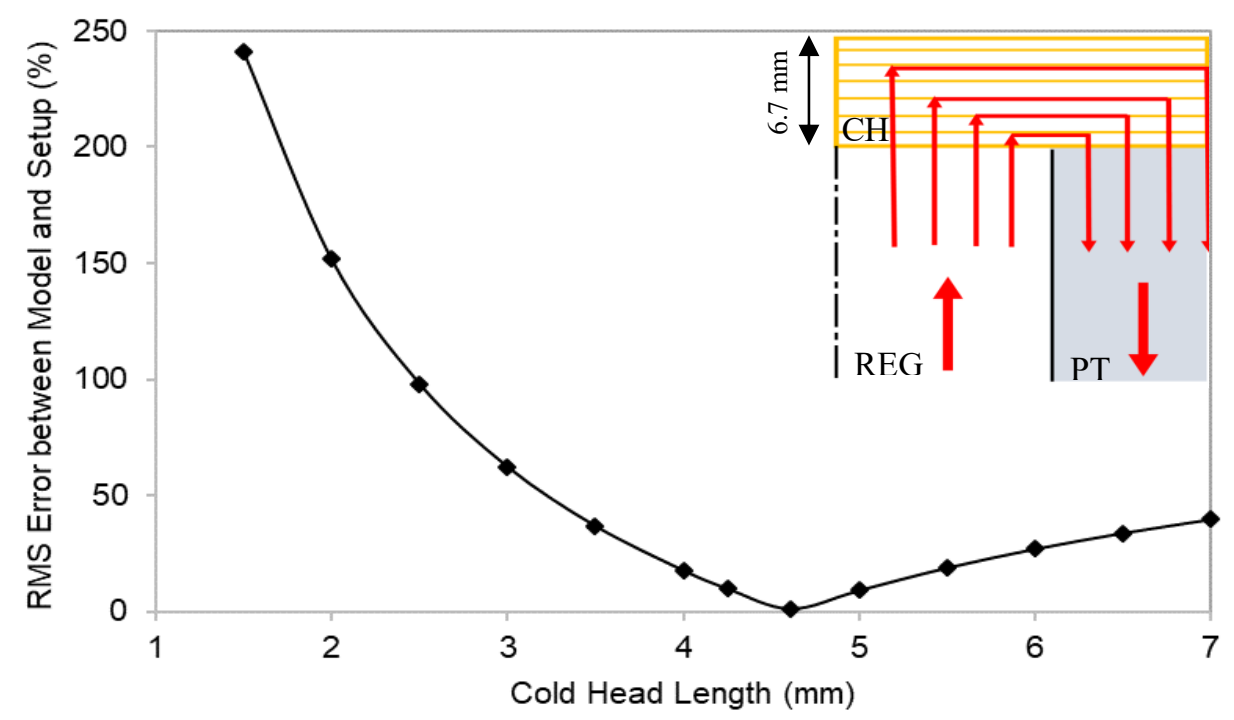

Figure 3: [Graph] The effective length of gas flow $(4.7 \mathrm{~mm})$ through the coaxial cold head is found to be $70 \%$ of the physical prototype length $(6.7 \mathrm{~mm})$ when minimizing RMS error between the model and flow test. [Top right] The axisymmetric cross-section of the coaxial flow through the cold head mesh (yellow lines). The dimensions that are true to the flow of the gas within the cold head are difficult to predict and model in Sage. 
The validated sub-models discussed in were implemented into a full coaxial cryocooler numerical model, with components modelled to numerically simulate mass flow and heat transfer throughout each unit indicated in Figure 1 and all connecting components. Figure 4 indicates the final temperature map of the full numerical model with the key components marked.

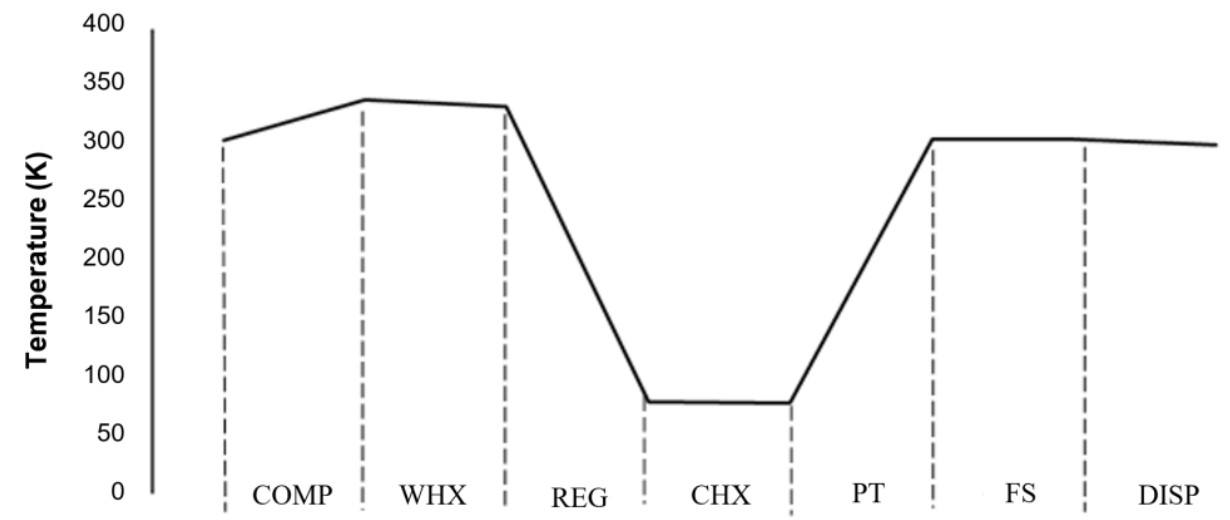

Figure 4: Temperature map of simulated gas through key components in the coaxial cryocooler model. COMP is the compressor unit, WHX is the heat rejector, REG is the regenerator, CHX is the cold head heat exchanger, PT is the pulse tube, FS is the warm end flow straightener, and DISP is the displacer.

\section{Simulated Cooling Performance at $80 \mathrm{~K}$}

The full model was used to predict the performance of the cryocooler at $80 \mathrm{~K}$ and compared with the numerical simulation results of the in-line cryocooler [2]. The parameters affecting the performance of the cryocoolers investigated were the operating frequency, the shaft power, the phase angle between the linear compressor and the displacer, and the regenerator and pulse tube component lengths. The inputs for each parameter were as shown in Table 2, which were kept constant throughout all parametric studies presented in this section, with the exception of the variable being investigated.

Table 2: Default inputs for the coaxial and in-line cryocooler numerical models. One parameter was varied in step intervals to address the effect it had on the overall 
performance of the cryocooler, whilst all others were kept constant at the values found in this table.

\begin{tabular}{cc}
\hline Input & Value \\
\hline $\begin{array}{c}\text { Compressor } \\
\text { stroke }\end{array}$ & $9 \mathrm{~mm}$ \\
$\begin{array}{c}\text { Displacer stroke } \\
\text { Operating } \\
\text { frequency }\end{array}$ & $4.8 \mathrm{~mm}$ \\
Phase angle & $60 \mathrm{~Hz}$ \\
Fill pressure & $41^{\circ}$ \\
\hline
\end{tabular}

\subsection{Effect of operating frequency on cooling power and pressure drop}

The operating frequency was varied to determine its effect on the cooling power of both cryocoolers at $80 \mathrm{~K}$, with all other inputs as per Table 2 . Figure 5 indicates that at higher frequencies, higher cooling powers are obtained and that the coaxial cryocooler is able to achieve a higher cooling power for a given frequency. A longer regenerator can permit a larger temperature difference to be generated at a given frequency, resulting in lower temperatures, but there are trade-offs between axial conduction, dead volume and the pressure drop. It is worth noting that the conductive losses estimated by the numerical models for both the coaxial and the in-line simulate very small conductive losses in the cryocooler, as Sage does not account for the geometric complexity of all the conductive surfaces. This no doubt results in an overestimation of the cooling power. 


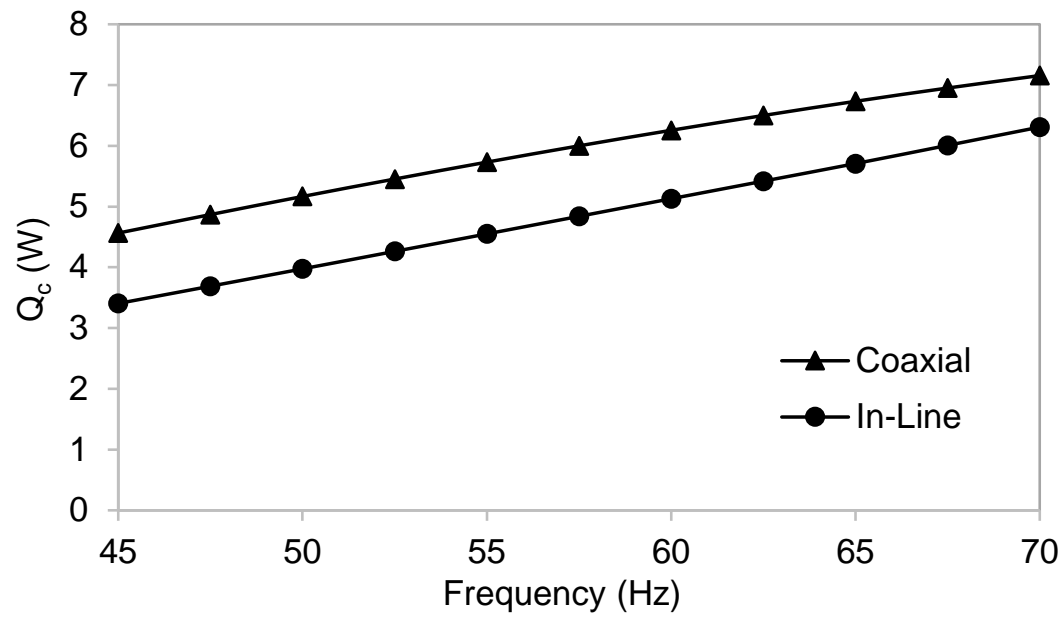

Figure 5: Cooling power $\left(Q_{c}\right)$ of the coaxial and in-line cryocoolers as a function of varying the frequency with Table 2 inputs.
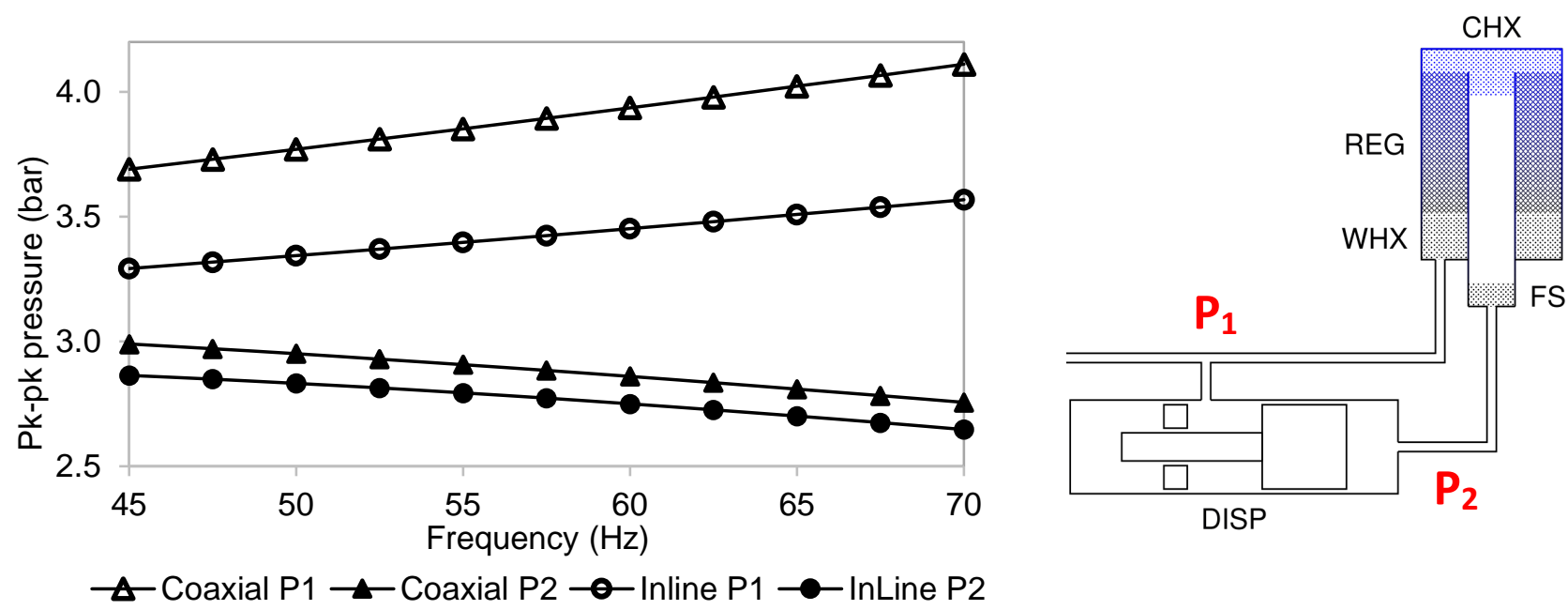

Figure 6: (Left) Effect of varying frequency on the pressure on either end of the coaxial and in-line cryocoolers and (right) the location of $P_{1}$ and $P_{2}$ in the coaxial cryocooler design, with Table 2 inputs. For both cryocoolers, $P_{l}$ is the pressure of the gas after the compression unit prior to entering the regenerator, and $P_{2}$ is the pressure of the gas after exiting the warm end heat exchanger. In this way, the difference between $P_{1}$ and $P_{2}$ indicates the pressure drop over the cryocooler.

The variation in pressure drop across the cryocooler as a result of varying operating frequency was also addressed as shown in Figure 6. The pressure drop across the cooler 
increases with an increase in operating frequency and the pressure drop across the coaxial pulse tube is larger than the in-line. Moreover, the pressure $P_{2}$ can be seen to decrease with an increase in operating frequency. As the pressure amplitude increases in the cooler pulse tube unit due to an increased operating frequency, this leads to a decrease in pressure at the back end of the displacer.

\subsection{Effect of shaft power on cooling power and efficiency at $80 \mathrm{~K}$}

The effect of the shaft power on the cooling power and efficiency of the cryocoolers was determined by varying the compression volume by changing the piston stroke. Figure 7 demonstrates the interplay between the two parameters for both the coaxial and in-line cryocoolers. Initially, the in-line cryocooler is able to achieve a higher cooling power for a given shaft power. After a crossover point at approximately $68 \mathrm{~W}$ of shaft power, the coaxial cryocooler outperforms the in-line in terms of cooling power. It is evident therefore that the in-line requires less volume flow rate to achieve $80 \mathrm{~K}$ cooling at the cold end of the cryocooler, whereas the coaxial cryocooler must be operated at higher volume flow rate to achieve the same target cooling. Figure 8 demonstrates that whilst the cooling power of the coaxial cryocooler is higher at high shaft powers, the efficiency is not necessarily greater than the in-line cryocooler. The relative Carnot efficiency is determined by the equation below, where $\eta_{r}$ is the relative Carnot efficiency, $W_{s h}$ is the total shaft power, $T_{h}$ is the warm end temperature $(300 \mathrm{~K})$ and $T_{c}$ is the cold end temperature $(80 \mathrm{~K})$.

$$
\eta_{r}=\frac{Q_{c}}{W_{s h}}\left(\frac{T_{h}}{T_{c}}-1\right)
$$




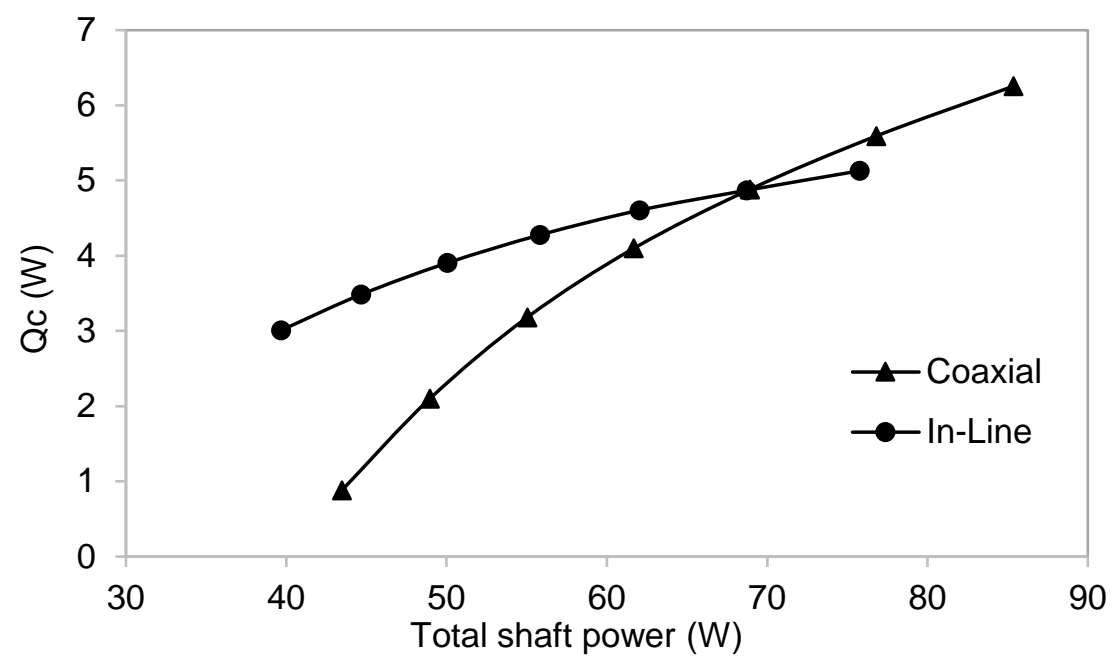

Figure 7: Effect of shaft power on cooling power as a function of compression volume, with Table 2 inputs.

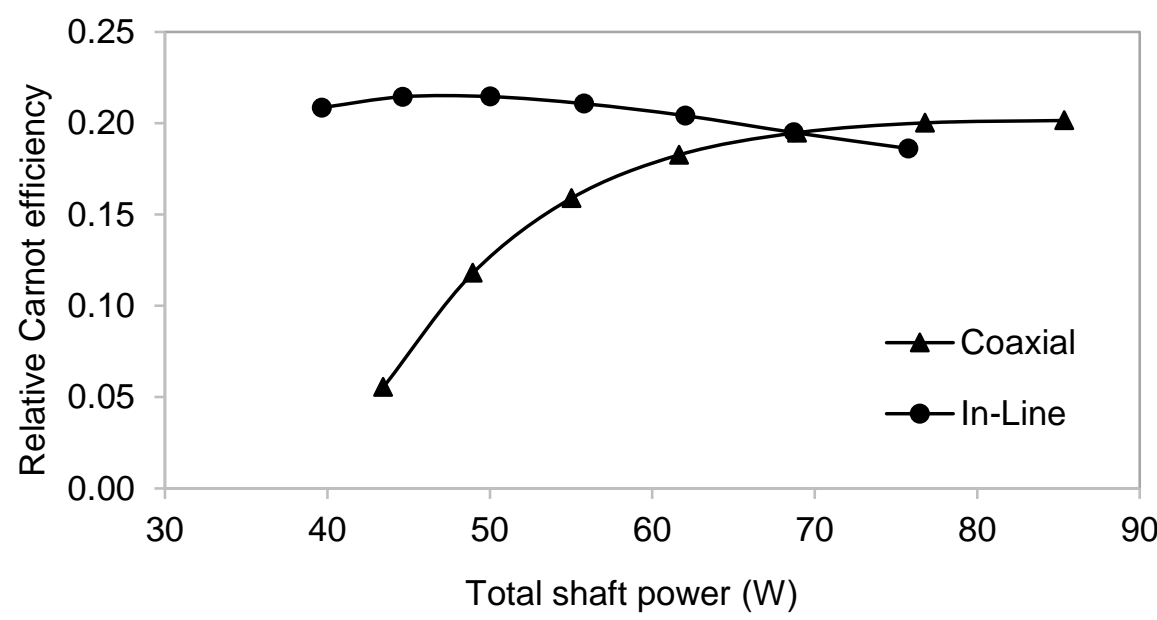

Figure 8: Relative Carnot efficiency of the coaxial and in-line cryocoolers with varying total shaft power by varying compression volume, with Table 2 inputs.

\subsection{Phase angle between linear compressor and displacer}

Figure 9 and Figure 10 show the effect varying the phase angle between the compressor and displacer has on the cooling power and the relative Carnot efficiency, respectively. It can be seen that higher cooling power is consistently achieved by the 
coaxial cryocooler, however, again, the efficiency achieved is not significantly better than the in-line cryocooler. The peak cooling power for both the coaxial and the in-line occurs at an optimum phase angle of $55^{\circ}$, whereas the relative Carnot efficiency reaches a maximum at a phase angle of $40^{\circ}$. Hence a trade-off between the two would need to be considered.

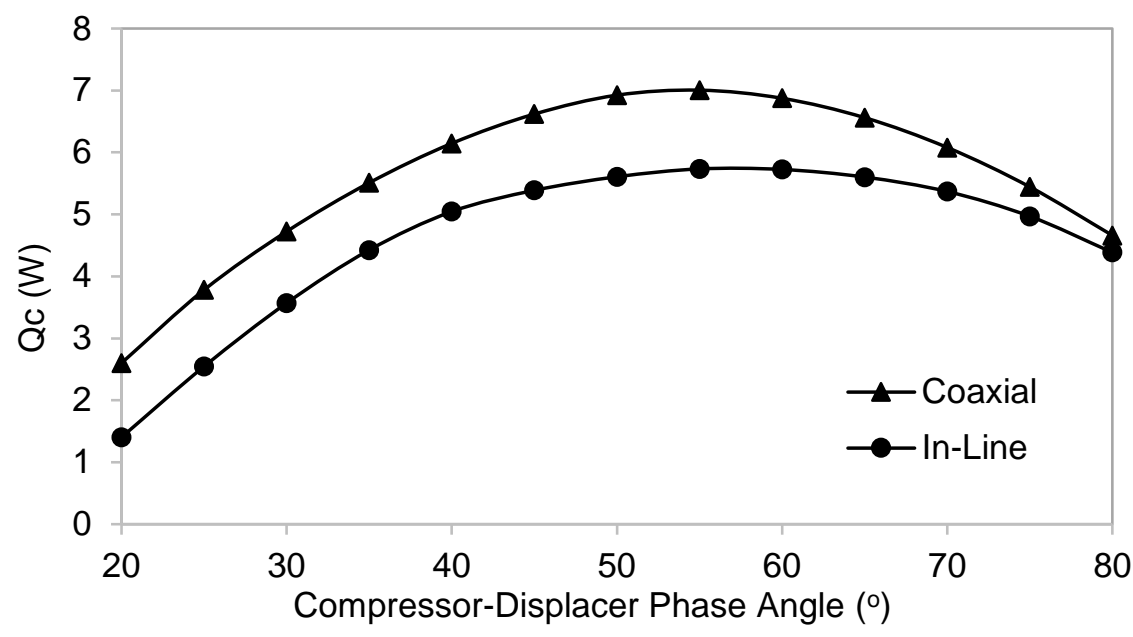

Figure 9: The effect of altering the phase angle between the linear compressor and displacer on the cooling power of the coaxial and in-line cryocoolers, with Table 2 inputs. 


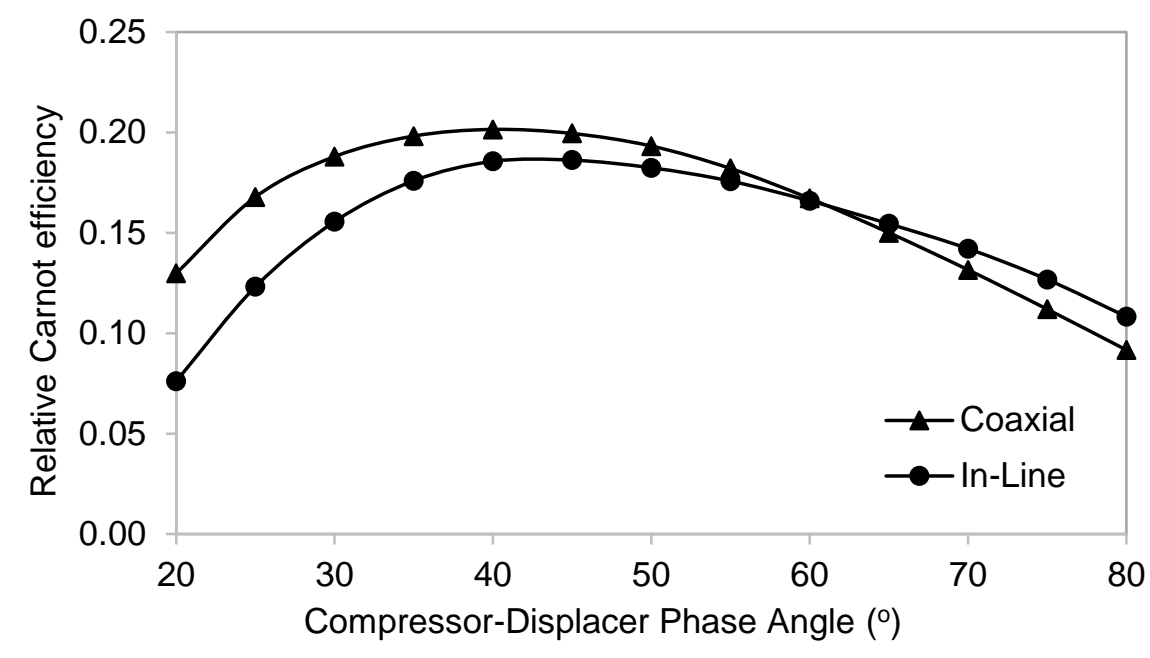

Figure 10: Change in relative Carnot efficiency of the in-line and coaxial cryocoolers as a result of altering the phase angle between the linear compressor and displacer, with Table 2 inputs.

\subsection{Performance sensitivity to pulse tube and regenerator length}
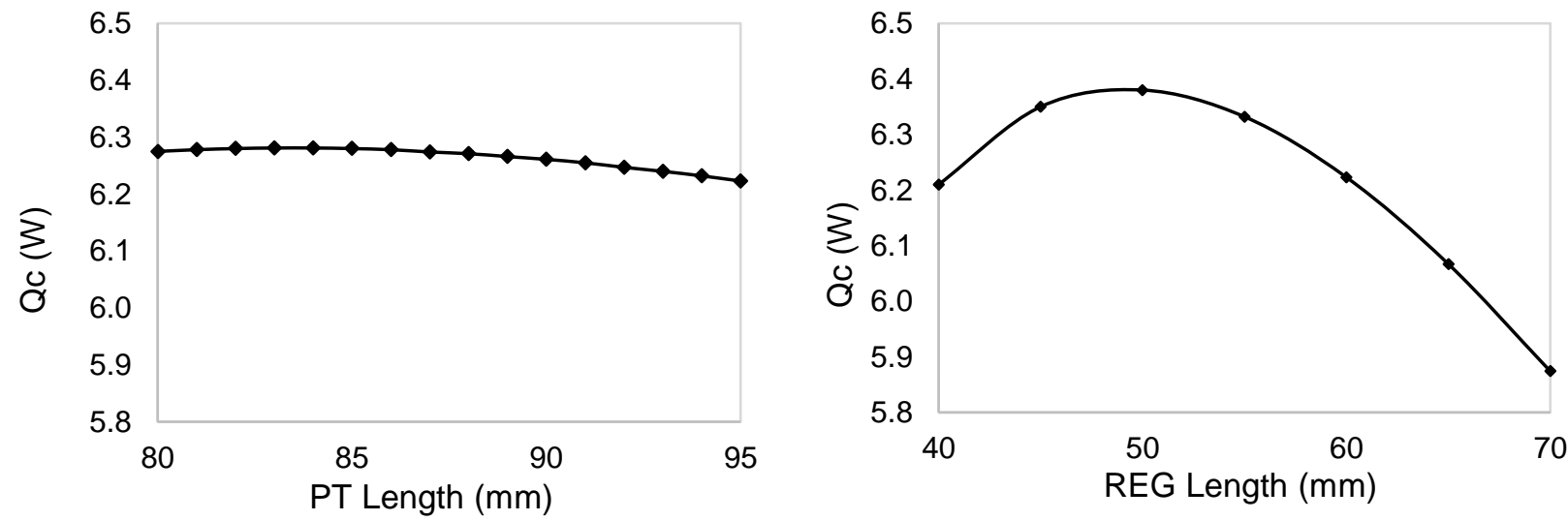

Figure 11: Change in cooling power $Q_{c}(W)$ as pulse tube length (left) and regenerator length (right) varies in the coaxial cryocooler, with inputs as per Table 2.

The numerical model can be used to determine the optimal length of the pulse tube and regenerator for highest net cooling power. Figure 11 indicates that the optimal 
regenerator length of $50 \mathrm{~mm}$ should be selected, and that the variation in the pulse tube length within this range only marginally affects the cooling performance.

The effect of the change in pulse tube and regenerator length on the variation in $\Phi_{p, P T}$, the phase between the pressure pulse along the pulse tube length, and $\Delta \mathrm{P}_{p k-p k, P T}$, the difference in peak-to-peak pressures on either end of the pulse tube, was investigated. The same was investigated for the regenerator. It was found that altering the pulse tube length does not greatly impact the $\Phi_{p, P T}$ and $\Delta \mathrm{P}_{p k-p k, P T}$, however, a noteworthy increase is observed in $\Phi_{p, R E G}$ and $\Delta \mathrm{P}_{p k-p k, R E G}$ with an increase in regenerator length, as shown in Figure 12.

A variation in $\Delta m_{p k-p k, P T}$, the difference in peak-to-peak mass flow, and $\Phi m_{, P T}$, the mass flow phase along the pulse tube, was observed by varying the pulse tube length, as shown in Figure 13. This sheds light on the physical impact of length variation on the mass flow in the pulse tube. In the regenerator, in addition to the increased difference in peak-to-peak pressures with a longer length, the mass flow phase increases also, as shown in Figure 13. This highlights these as the factors responsible for the influence on the variation in cooling power with component length, presented in Figure 11.

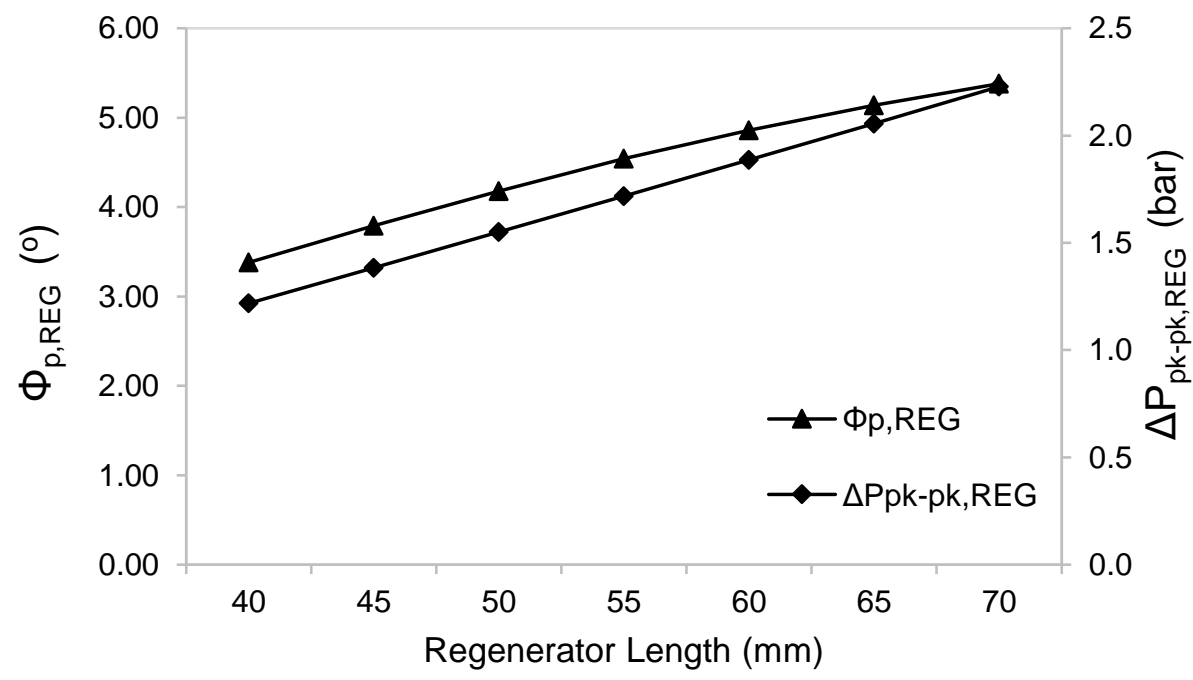

Figure 12: Variation of in $\Phi_{p, R E G}$ and $\Delta \mathrm{P}_{p k-p k, R E G}$ with an increase in regenerator length, with all other inputs as per Table 2 . 

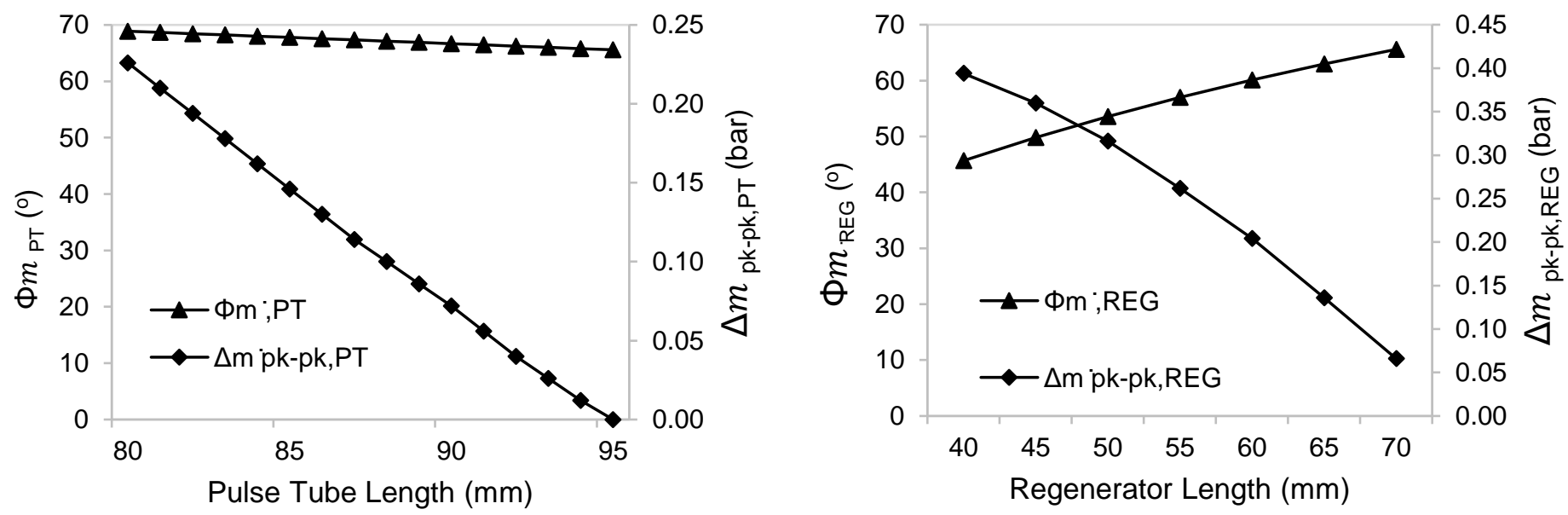

Figure 13: Variation of $\Phi \boldsymbol{m}$ and $\Delta \boldsymbol{m}_{p k-p k}$ for the pulse tube (left) and regenerator (right).

\subsection{Towards a passive displacer design}

The desire to reduce additional moving parts and reduce the complexity of the cryocooler design would improve the reliability and longevity of a cryocooler selected for a spacecraft mission. Whilst inertance tubes succeed in tackling this problem, its use does not allow for power to be recycled back into the cryocooling system in the manner that a displacer does. A potential solution is to employ a passive displacer. The further challenge, however, would be to carefully design the correct dynamics of the displacer to achieve a high efficiency similar to the active displacer, as has been numerically simulated in this paper and previously experimentally demonstrated [2] [4] [5] [6] [11]. A damper will likely be required to tune the phase angle, which will undoubtedly add an additional loss term due to its energy dissipation. Work by Wang et al. [12] have achieved a relatively high Carnot efficiency by using a rod-less displacer.

Further analysis, shown in Figure 14, of the numerical performance data obtained for the coaxial and the in-line cryocoolers with active displacers demonstrated that the displacer shaft power computes to give negative values at lower phase angles. These results are useful in shedding light on the conditions in which the displacer can be 
driven passively. This will provide useful starting inputs for designing the damping parameters and sizing of a displacer to achieve the correct dynamics for passive operation. This will require the compressor to operate at resonance and the displacer to operate near resonance, in order to achieve the correct phase angle.

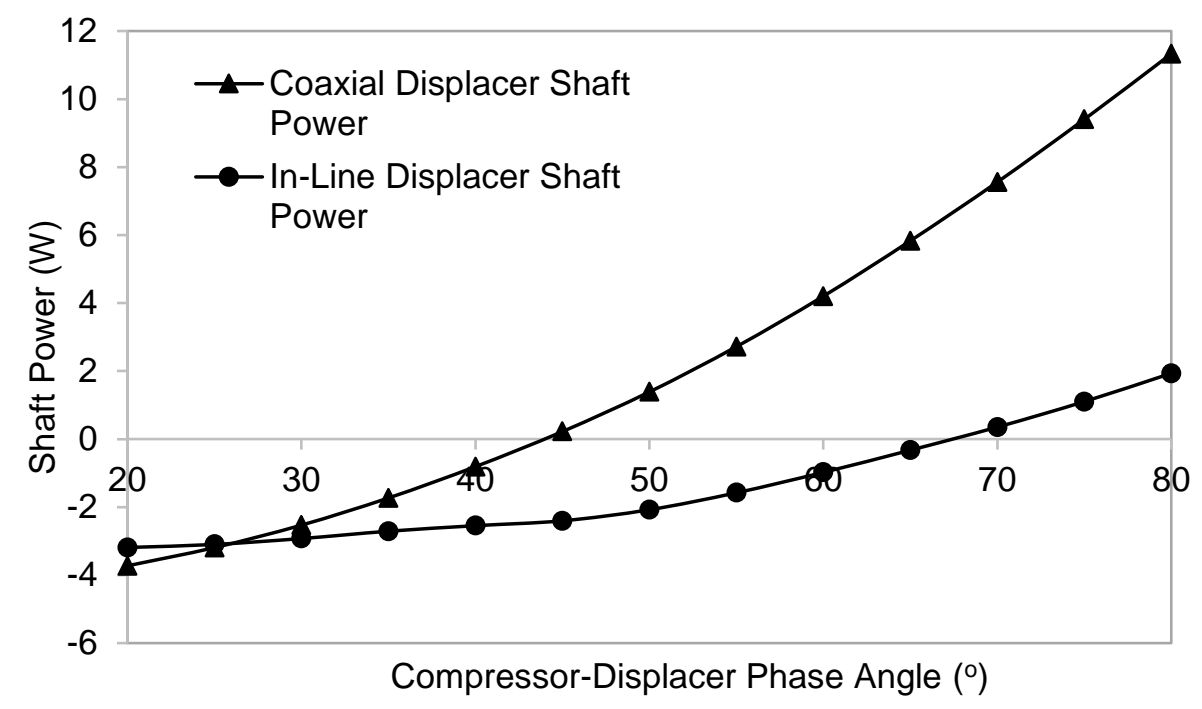

Figure 14: Displacer shaft power with varying compressor-displacer phase angle, with all other inputs as per Table 2 .

\section{Conclusions}

In conclusion, a numerical model has been devised for a complete $80 \mathrm{~K}$ SPTC with an active displacer in a coaxial configuration. Flow tests were completed on selected sub-assemblies such as the coaxial cold head to provide starting inputs for the simulation. The coaxial cryocooler numerically simulates $6 \mathrm{~W}$ of cooling at $80 \mathrm{~K}$ with an input power of $85 \mathrm{~W}$, at a fill pressure of $28 \mathrm{bar}$, an operating frequency of $60 \mathrm{~Hz}$ and a compressor-displacer phase angle of $41^{\circ}$. The numerical performance indicates that the coaxial cryocooler may outperform the in-line cryocooler in terms of cooling 
power, but present no particularly significant improvement in terms of efficiency. These numerical findings are due to be compared with experimental findings that are currently underway. These findings can be used to inspire the development of a passive displacer SPTC.

\section{Acknowledgements}

The authors would like to acknowledge partial funding from the EPSRC with EP/N017013/1 and the support of Honeywell Hymatic for this project and in the broader activities pertaining to SPTCs within the Cryogenic Engineering group at the University of Oxford. Furthermore, they would like to thank the CSA/NASA committee and all those involved in the review of Cryogenics.

\section{References}

[1] P. De Boer, "Performance of the inertance pulse tube," Cryogenics, vol. 42, no. 3, pp. 209-221, 2002.

[2] M. A. Abolghasemi, K. Liang, R. Stone, M. Dadd and P. Bailey, "Stirling pulse tube cryocooler using an active displacer," Cryogenics, vol. 96, pp. 5361, 2018.

[3] S. Zhu and M. Nogawa, "Pulse tube stirling machine with warm gas-driven displacer," Cryogenics, vol. 50, pp. 320-330, 2010.

[4] Y. Shi and S. Zhu, "Experimental investigation of pulse tube refrigerator with displacer," International Journal of Refrigeration, vol. 76, pp. 1-6, 2017.

[5] H. Dang, "40 K single-stage coaxial pulse tube cryocoolers," Cryogenics, vol. 52, pp. 216-220, 2012.

[6] N. Wang, M. Zhao, Y. Ou, Q. Zhu, L. Wei, H. Chen, J. Cai and J. Liang, "A high efficiency coaxial pulse tube cryocooler operating at $60 \mathrm{~K}$," Cryogenics, vol. 93, pp. 48-50, 2018.

[7] J. M. Duval, I. Charles, C. Chassaing, J. Butterworth, G. Aigouy and J. Mullie, "15 K pulse tube design for ECHO mission," in AIP Conference Proceedings 1573, 533, 2014. 
[8] D. Gedeon, "Sage: object-oriented software for cryocooler design," Cryocoolers 8, pp. 281-292, 1995.

[9] D. Gedeon, "DC gas flows in Stirling and pulse-tube cryocoolers," Cryocoolers 9, pp. 385-392, 1997.

[10] Y. Zhao and H. Dang, "CFD simulation of a miniature coaxial Stirling-type pulse tube cryocooler operating at $128 \mathrm{~Hz}$," Cryogenics, vol. 73, pp. 53-59, 2016.

[11] Y. Zhang, H. Li, X. Wang, W. Dai, Z. Yang and E. Luo, "Advances in a high efficiency commercial pulse tube cooler," in IOP Conf. Series: Materials Science and Engineering 278, 2017.

[12] X. Wang, Y. Zhang, H. Li, W. Dai, S. Chen, G. Lei and E. Luo, "A high efficiency hybrid Stirling-pulse tube cryocooler," AIP Advances, vol. 5, no. 3, pp. (037127)1-5, 2015. 